When asked who they thought arranged part-time training posts, $14(31 \%)$ were unsure; $1(2 \%)$ replied no-one; $5(11 \%)$ replied trainees themselves; $5(11 \%)$ erroneously stated some other person, and only 16 $(36 \%)$ correctly suggested the clinical dean or the regional health authority.

Nine respondents made additional comments about part-time training, and these fell into three categories. The first revealed fundamental misunderstandings about the current availability and nature of part-time posts. For example, that part-time posts did not involve training or were not recognised by the Royal College of Psychiatrists. One respondent replied that he was unaware any part-time posts were available at all.

Another category of comments were swingeingly critical of the current arrangements, notably that part-time should really mean that, and not "a parttimer trying to do a full-time job". One respondent had tried for a supernumerary post but been unable to obtain one because of lack of funds for the PM(79)3 scheme. A few comments were made about current part-time posts being "inferior". Several subjects also commented that such posts were available only for women, especially after they had had children, and that part-time posts should be accessible for men too. One commented that "jobs ... done mostly by women are automatically seen as inferior and not serious".

Further comments concerned the feasibility of part-time work; one trainee foresaw a possible danger in creating part-time posts if they were used to replace full-time posts. Another respondent suggested that the College and health authorities should be more amenable towards flexible working arrangements.

\section{Comment}

This survey shows several interesting aspects to attitudes held by junior psychiatric trainees to part-time training. There is widespread confusion about what is on offer, but those who do know what there is consider it to be far from satisfactory. This is perhaps not surprising since all those polled were working full-time, and had not opted for part-time work for some reason. What is surprising, however, is that $25 \%$ of these full-timers have in the past wanted to work part-time yet not been able to do so. Sixty-four per cent wished to work part-time in the future, and while being married made such a desire more likely, it is interesting to note that there was no significant difference here between men and women.

It seems clear that the stereotype of a supernumerary part-time post for female trainees with young families is not what the current generation of psychiatric trainees want, or indeed believe should be on offer. Furthermore, the consensus view is that the current provision of part-time posts is qualitatively and quantitatively inadequate.

The need for a review of part-time training in particular for SHOs and registrars, has already been recognised (Hinchcliffe, 1990). It is to be hoped that the opinions of such potential trainees will be taken more into account in the future.

\section{References}

HinchClifFe, M. (1990) Flexible opportunities for parttime training in psychiatry (letter). Psychiatric Bulletin. $14,677$.

RHODES, P. (1990) Medical women in the middle: family or career? Health Trends, 22, 33-36.

\title{
The physical examination in psychiatry
}

\author{
StePHEN Hughes, 31 Roseview Crescent, Kinmel Bay, Clwyd LL18 5BY
}

There are many studies in the medical literature attempting to confirm the value of a comprehensive physical examination in the psychiatric in-patient population. An appraisal of the literature and review of psychiatric admissions in a large teaching district offers some evidence to the contrary.

Many papers have focused on the incidence of significant physical illness in psychiatric in-patients (Koran et al, 1989; Merridge, 1960; Snaith \& Jacobson 1965). Unfortunately these estimates are often based on figures over 20 years old. With a greater emphasis on "community care" it may be suspected that today's psychiatric in-patient population differs in some respects from that of the 1960 s.

The prevalence of physical illness said to be directly "causal" to an episode of psychiatric illness has been consistently estimated at $5-8 \%$ of in-patients in separate studies. The overall prevalence estimates vary more; between 15-50\% (Merridge, 1960; Snaith \& Jacobson, 1965; Koran et al, 1989). One question this paper attempts to answer is how much unknown physical illness is discovered on routine physical 
examination, as performed on a daily basis, in an English teaching hospital group?

\section{The study}

The psychiatric casenotes of all admissions to the three Leicester psychiatric teaching hospitals during July-August 1990 were assessed. An age limit of 18-75 was imposed and all cases of proven dementia excluded. Only those admissions which resulted in a full in-patient episode of at least one week were included, as this would give sufficient time for a full physical screen to be completed if required by the admitting team. A review of all detected pathology was correlated with the initial recorded physical examination and subsequent investigations.

The quality of the physical examination was assessed by simple comparison of standard features, such as whether blood pressure measurement or examination of gait or of cranial nerves were undertaken.

\section{Findings}

The casenotes of 178 in-patients were reviewed. Of these 51 were of new patients and 127 were of re-admissions. There were 77 males and 101 females. Of these, 26 patients were not examined at all during their in-patient stay (15\%), including four new in-patients!

Of those patients examined, comprehensiveness of examination varied considerably. The only new pathology found on initial assessment was of one raised blood pressure reading in a 55-year-old man.

After review of all in-patient episodes, the only medical problems elicited were: one breast lump, discovered by the patient; one case of Wernicke's encephalepathy in a 29-year-old man admitted with confusion and gait disturbance and one chest infection in an elderly man with a temperature and cough. One elderly lady had a mild asymptomatic hyperthyroidism and another had a recurrent symptomatic urinary tract infection. A known non-insulin elderly diabetic had moderately raised blood sugars.

In no cases did treatment of the medical symptoms alter the eventual psychiatric management, except for the patient with Wernicke's encephalepathy.

\section{Comment}

Many psychiatrists choose to ignore the physical examination. In a survey of American psychiatrists by Krummel \& Kathol (1987) 63 out of 123 respondents said they never performed a physical examination on any of their patients. (Although 92\% of in-patients had a "routine" laboratory screen).

Even a large recent survey from America quoting an incidence of $45 \%$ "important physical disease" overlooked the fact that virtually all cases of illness contributing to psychiatric disorder were of known organic brain disease (Koran et al, 1989). The author's definition of "important" disease included migraine, premenstrual tension and irritable bowel syndrome. The three other cases of illness contributing to psychiatric disorder were all amenable to laboratory screening, namely anaemia, hypothyroidism and hyperparathyroidism.

This survey utilised the findings of 26 different psychiatric trainees in teaching posts, none of whom were aware that their work was being analysed. It would be naive to think that these results are particularly worse than in many other hospitals.

The main finding in this survey is the poor standard of performing and recording physical examination. Rigby \& Oswald (1986) have looked in detail at the examination of 100 consecutive psychiatric admissions. The important missed clinical findings were of cases of drug induced Parkinsonism and tardive dyskinesia.

It is worth noting that nearly all cases of important remediable pathology were found through careful examination of the nervous system. This was the area of physical examination most poorly conducted in this survey. The nervous system was not examined at all in 41 out of 178 patients $(23 \%$ ) and thoroughly in only 13 cases $(7 \%)$.

\section{References}

Koran, L. M., SuX, M. C., Marton, K. I. el al (1989) Medical evaluation of psychiatric patients. Archives of General Psychiatry, 46, 733-740.

Krummel, S. \& Kathol, R. G. (1987) What you should know about physical evaluations in psychiatric patients. General Hospital Psychiatry, 9, 275-279.

MERRIDGE, C. G. (1960) Physical disorders in psychiatric illness. Lancel, $i i, 949-951$.

RugBY, J. C. \& Oswald, A. G. (1986) An evaluation of the performing and recording of physical examinations by psychiatric trainees. British Journal of Psychiatry, 150, 533-535.

SNAIT, R. P. \& JACOBSON, S. (1965) The observation ward and the psychiatric emergency. British Journal of Psychiatry, 111, 18-26. 